\title{
RESTORATION AND SPARE CAPACITY ASSIGNMENT IN WDM NETWORKS
}

\author{
B. Van Caenegem*, F. Deturck and P. Demeester \\ Dep. of Information Technology, Univ. of Gent - IMEC
}

technical subject area:

designing WDM networks for reliability and availability.

keywords:

spare capacity planning + strategies for network restoration, optimisation techniques (integer linear programming)

\section{Extended abstract}

WDM technology is an emerging technology to enhance the capacity of the existing fiber plant. The throughput is in the order of tens or hundreds of Gbit/s, even in the order of Tbit/s [1,2]. This urges on finding adequate and cost effective means to survive failures in the network. In this paper restoration techniques in WDM networks are discussed and their spare capacity requirement is compared.

*supported by a grant of the National Fund for Scientific Research of Belgium 
Only single link failures are considered as in large scale networks a cable break or line system failure is supposed to happen more likely than a node failure. Besides in a node vital parts can be protected by duplicating some equipment. Note that a line system termination is physically located in a node, but its failure affects the link and does not affect the node!

Three restoration strategies are considered: link restoration, path restoration and path restoration with link-disjunct route (or the last one also called backup path restoration). Restoration is understood as rerouting through a reconfiguration of crossconnects. In WDM networks we distinguish wavelength routing networks and wavelength translating networks. In wavelength translating networks the crossconnects are equipped with wavelength converters and therefore there is no constraint on the wavelengths used by the restoration route. The signal may be routed on different wavelengths in the sequential links of the route. In wavelength routing networks however, there are wavelength constraints on the restoration routes. In case of link restoration only the channel in the interrupted link which might be part of a route, is rerouted and must therefore be rerouted on the same wavelength as the rest of the path.

In case of path restoration the transmitters can be tunable or spare transmitters on other wavelengths are available. In that case a route on another wavelength is allowed for rerouting, otherwise a route on the original wavelength must be found. It is clear that the same wavelength must be available along the entire route as there is no possibility of wavelength conversion in the intermediate nodes.

To be able to reroute, spare capacity must be assigned in the network. This spare capacity assignment for each of these strategies is performed by using Integer Linear Programming (ILP) [3,4]. The problem is therefore expressed in a linear model. Linear Programming (LP) finds a lower bound for the specified problem and 
an interrupted branch and bound process returns an integer solution which is a rounded solution of the LP-solution.

In terms of spare capacity requirement, link restoration is most expensive, but when implemented as restoration technique in a network, it allows a fast rerouting upon a failure. Path restoration is cheaper but the restoration process takes longer as the interrupted paths upon a failure are rerouted separately. Releasing capacity of interrupted paths on intact links allows capacity reuse on this links and therefore less spare capacity to be fully restorable. However, the drawback is that it requires more reconfiguration actions in the network.

The third studied restoration technique, path restoration with linkdisjunct route, uses for each path one restoration route that can be used in case of a breakdown of the working path due to any link failure. By optimising the choice of the restoration route per path, this technique requires less spare capacity than link restoration and only slightly more than path restoration. However, it is simpler to implement than path restoration as only one restoration route must be stored for each path.

The remarks above hold for wavelength translating networks. It is expected that they do as well for wavelength routing networks. The benefit of wavelength conversion for static routing was already found to be low [5]. For the spare capacity assignment and rerouting problem first results show that we can expect to come to the same conclusion. 


\section{References}

[1] H. Onaka et al., "1.1 Tb/s WDM transmission over $150 \mathrm{~km}$ $1.3 \mu \mathrm{m}$ zero-dispersion single-mode fiber," Conference on Optical Fiber Communications (OFC'96), San Jose, CA, February 26 - March 1, 1996, post-deadline paper PD19.

[2] Y.Yano et al., "2.6 Terabit/s WDM Transmission Experiment using Optical Duobinary Coding", ThB.3.1, ECOC'96, Oslo, Sept. 16-19 1996.

[3] M. HERZBERG, "A Decomposition Approach to Assign Spare Channels in Self-Healing Networks", GLOBECOM '93, pp 1601-1605, 1993.

[4] B.Van Caenegem, N. Wauters, P. Demeester, "Spare Capacity Assignment for Different Restoration Strategies in Mesh Survivable Networks”, ICC'97, Montreal, Canada, June 1997

[5] N. Wauters, P. Demeester, "Design of the Optical Path Layer in Multiwavelength Cross-Connected Networks", IEEE Journal on Selected Areas in Communications, Vol. 14, NO. 5, June 1996 